\title{
Implementation of Hardware-in-the-Loop Based Platform for Real-time Battery State of Charge Estimation on Li-Ion Batteries of Electric Vehicles using Multilayer Perceptron
}

\author{
Süleyman Çeven ${ }^{1}$, Raif Bayır*2
}

\begin{abstract}
Submitted: 21/10/2020 Accepted : 08/12/2020
Abstract: In this study, hardware-in-the-loop based real-time state of charge estimation was performed in Li-Ion batteries, which are widely used in hybrid and battery electric vehicles. The state of charge is estimated on the Li-Ion battery cell that forms the electric vehicle battery system. Multi-layer perceptron approach has been preferred as a method for estimating the battery state of charge. Discharge experiments based on different electrical loads were applied to the Li-Ion battery cell to be used in multilayer perceptron learning processes. An experimental setup has been prepared to perform the discharge process under different electrical loads. In each discharge experiment, battery open circuit voltage, battery discharge current and battery cell temperature parameters were measured and were recorded. By using the data obtained from the experiments on the battery cell, a multilayer perceptron model was created in MATLAB environment. After creating the multilayer perceptron model, the real-time battery state of charge the was estimated at different discharge currents in the experimental setup and the results obtained were evaluated.
\end{abstract}

Keywords: Li-Ion batteries, hardware-in-the-loop, electric vehicles, multilayer perceptron, artificial neural networks, state of charge estimation

\section{Introduction}

Due to the decreasing fossil fuel reserves in the world and the increase in $\mathrm{CO}_{2}$ emissions released into the atmosphere, there has been a tendency to seek new resources, use sustainable and renewable energy in today's automotive industry [1]. Increase of new studies in the automotive sector shows that Battery Electric Vehicles (BEVs) and Hybrid Electric Vehicles (HEVs) have been preferred as a solution of these problems [2]. Generally, Electric Motor (EM) is used in BEVs, on the other hand Internal Combustion Engine (ICE) and EM together are used in HEVs [3], [4]. The widely using of BEVs and HEVs has led to an increase in studies on batteries [5]. Today, various battery types such as leadacid batteries, Lithium-ion batteries, Sodium-Sulfur batteries, Nickel-Cadmium batteries are used in these technologies [6]. The most used type of battery in BEVs and HEVs is Lithium-based batteries due to their high energy density, durable structure, fast charging, low maintenance costs, and long life [7], [8]. However, such batteries can easily break down in cases of incorrect charging and discharging due to their chemical structure [9]. Therefore, in lithium-based batteries, the charging and discharging processes must be performed by monitoring the parameters in the battery.

The State of Charge (SoC) in the batteries is the most important parameter that shows the energy capacity of the battery [10] - [12]. Knowing the SoC of a battery correctly and without error enables the Battery Management System (BMS) to work correctly and protect the battery from overcharging, discharge and heating [13]. Today, unfortunately, SoC cannot be measured directly using sensors [14], [15]. For this reason, some estimation methods

${ }^{1}$ Department of Electronics \& Automation Technology, Duzce Vocational School, Düzce University, 81010- Turkey ORCID ID : 0000-0002-8970-4826

${ }^{2}$ Department of Mechatronics Engineering, Faculty of Technology,

Karabuk University, 78100- Turkey

ORCID ID : 0000-0003-3155-8771

* Corresponding Author Email: rbayir@karabuk.edu.tr should be used to measure the SoC [12], [16]. Today, methods and algorithms such as direct measurement methods, Artificial Intelligence (AI) methods, model-based methods and hybrid methods in battery SoC estimation are available in the literature [11] - [15], [17] - [19]. It describes three basic methods such as direct measurement methods, Coulomb counting method [20][25], electrochemical impedance spectroscopy method [26]- [31], battery Open Circuit Voltage (OCV) based methods [32]- [36]. With direct measurement methods, the instantaneous measured parameters of the battery are used to estimate the battery SoC [37]. However, since the initial parameters of the battery cannot be estimated with these methods, it is necessary to know whether the battery is fully charged or completely empty for accurate SoC estimation. Therefore, it can be said that these methods are poor in terms of accuracy [16].

In addition to direct measurement methods, indirect measurement methods are also used in estimating the battery SoC [38]. Adaptive filter-based methods, model-based methods [39] - [42], adaptive Artificial Intelligence (AI)-based methods [43] - [47] and hybrid methods [48] - [50] are used as indirect measurement methods [51]. Unlike direct measurement methods, indirect measurement methods yield predictive results with higher accuracy [16]. Classification of the methods used today in the SoC estimation in batteries and the disadvantages of these methods are given in Figure 1.

Many studies have been carried out on the estimation of SoC, and a new one is added to the studies on this subject every day. The correlation between OCV and SoC is often used in SoC calculation algorithms. However, the relationship between OCV and SoC is not the same for each battery. The SoC and capacity of the Lithium-Ion (Li-Ion) battery is estimated using the double Extended Kalman Filter (EKF) and SoC-OCV relation. According to the experimental results, this method works with an error of less than 5\% [17]. In a study on the methods and applications of SoC 
determination, it was emphasized that the operating conditions from battery to battery will be different, so the method of estimating the battery SoC may be suitable for some batteries or not for others. For example, batteries used in solar energy storage, batteries used in electric vehicles and batteries used in telecommunication be different from each other [53]. In battery SoC estimation, electrical circuit model is widely used due to ease of calculation. However, the SoC estimation performance of this method is low and the modeling time is short [54]. Using adaptive iterative Artificial Neural Network (ANN), SoC estimation in LiIon batteries yields successful results [46]. In a study using ANN and $\mathrm{EKF}$ in the SoC estimation of Li-Ion batteries, electrical measurement data during the charging or discharging process are used to train the neural network [17]. In the estimation of battery SoC, the combination of Genetic Algorithm (GA)-ANN yields efficiently results avoid from complex mathematical operations [55]. It is claimed that the artificial intelligence approach is efficient and can predict SoC in advance [56]. The ANN algorithm is used to estimating the SoC value with the discharge test [47]. In this study, the SoC estimation of Li-Ion battery cell, which is widely used in BEVs and HEVs today, was performed using a Multi-Layer Perceptron (MLP).

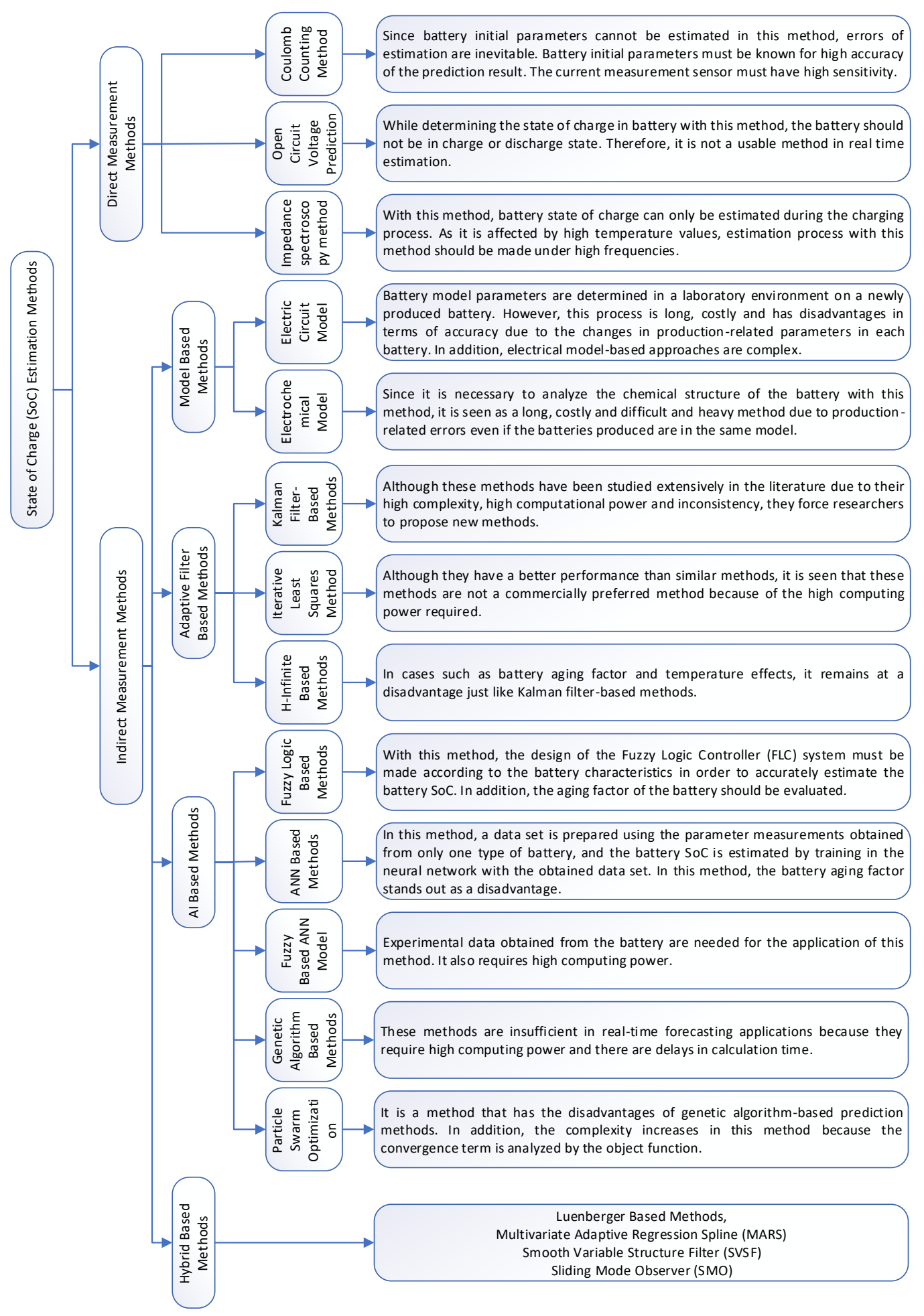

Fig. 1. Battery SoC estimation methods and disadvantages [52] 


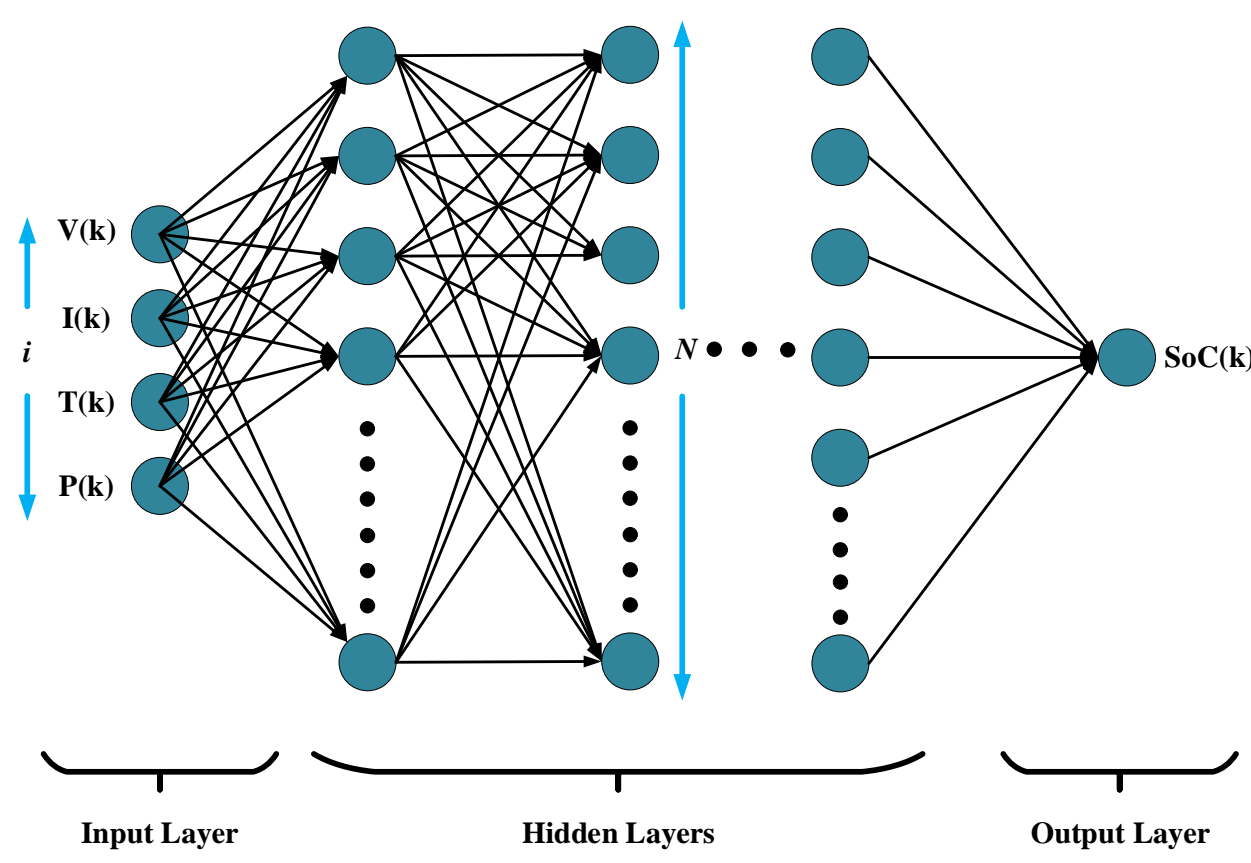

Fig. 2. Multilayer perceptron network model for battery SoC estimation.

The battery temperature, OCV, current and instantaneous electrical power parameters used in the training of the MLP were obtained by the discharge process on different electrical loads on the battery with an experimental setup. A programmable load device was used to discharge the battery, and a Li-Ion battery charger was used to charge the battery. A temperature sensor was used for battery temperature measurement, a voltage sensor for OCV measurement, and a current sensor for current measurement. Battery parameters obtained from the sensors were monitored in real-time with the experiment control card and recorded on the Secure Digital (SD) memory card. At the same time, the battery $\mathrm{SoC}$ was calculated in real-time with the Coulomb Counter method on the experiment control card and recorded on the SD memory card. MLP designed in Matlab environment was trained with the part of the data obtained from discharge experiments reserved for training. After the network training, the MLP performance was tested and the results were interpreted.

In the second part of the article, modeling of MLP, in the third part, the Real-time SoC estimation using MLP, in the fourth part the results and discussions are given.

\section{Modeling a Multi-layer Perceptron}

MLPs are one of the artificial intelligence methods in the sub-class of machine learning used in different fields such as system modeling, anomaly detection, classification applications [57]. The MLP consists of a series of structures called neurons that mimic the information processing and information acquisition capabilities of the human brain. MLPs are frequently used especially in modeling processes of systems with complex mathematical models [44]. MLPs work with the principle of transmitting the input parameter values to the output by connecting layers consisting of a series of neurons among themselves. In the process of transferring input parameters to the output, the values transmitted to individual neurons in each layer are mathematically processed with different weights and threshold values in the neurons, and then transmitted to the next layer. Thus, complex system models can be easily adapted using MLP [58]. MLPs can perform learning and updating processes in their structures in order to adapt to the system they are adapted. MLPs need data in accordance due to their working principles. Without the need for detailed physical parameters related to a complex system, they create a highaccuracy model of the system by imitating the responses of the system according to certain input variables. Thus, they take their place in the literature as one of the best methods of avoiding the difficulties and computational complexity encountered in the process of mathematical modeling of systems [59].

\subsection{Multi-layer Perceptron Model}

The MLP structurally consists of the input layer, at least one hidden layer consisting of nodes to similar the relationship between input and output layers, and the output layer. In Figure 2, the MLP structure used in the estimation of SoC of the Li-Ion battery is given. The network inputs consist of the current, voltage, temperature and electrical power parameters obtained from the battery discharge experiments, while the MLP output consists of the battery SoC parameter obtained by the Coulomb count method during the battery discharge experiments.

In the MLP structure given in Figure 2, each layer is connected to each other through nodes. There are special weight values and a threshold value parameter for each input parameter on the neurons forming the layers in the neural network. After the weight values and neuron threshold value parameters are processed in the neuron, the results of the mathematical operation are applied to the activation function defined for each node output. Hyperbolic tangent sigmoid function is generally used as activation function in MLP structures. The hyperbolic tangent sigmoid function is given in Eq. (1). The neuron outputs in the layers are mathematically given in Eq. (2).

$$
\begin{aligned}
& \mathcal{F}_{\text {tansig }}(u)=\frac{2}{1+e^{-2 u}}-1 \\
& y_{j}=\mathcal{F}\left(u_{j}\right)=\mathcal{F}\left(\sum_{i} \omega_{i j} x_{i}+b_{j}\right)
\end{aligned}
$$

Here, $x_{i}$ represents the output of node $\mathrm{i}$ of the layer before the relevant neuron, $\omega_{i j}$ represents the weight of the connection between node $j$ of the current layer from node $i$ of the layer before the neuron, and $b_{j}$ represents the threshold value of node $\mathrm{j}$. The weight and threshold values defined for each node and connection in the hidden layer and output layers of the neural network are 
determined by the training data of the network. In this study, Back Propagation (BP) algorithm was used to adjust weight and threshold parameters. During the training process of the network, the BP algorithm transmits the error value between the output parameter and the output parameter of the network to hidden layers, and operates according to the principle of iterative updating of weight and threshold parameters and minimization of error owing to the learning function. For detailed information about the BP algorithm, see. [60]. Figure 3 gives an example of a neuron.

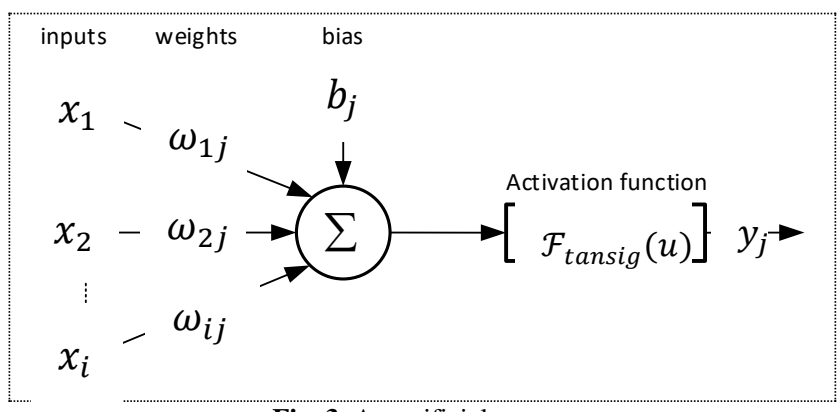

Fig. 3. An artificial neuron

\subsection{Preparation of Training Data}

In BEVs and HEVs, the most important process in MLP and SoC estimation in their batteries is the preparation of the data required for training the neural network. The situation that should be taken into consideration in the preparation process of the training data of the network is that BEVs and HEVs are exposed to variable loads in their real-life use. Changes in electrical loads vary according to road conditions, vehicle speed and driving style. Therefore, in order for the network to have high prediction accuracy, the variable parameters of the data used in the training of the network should include the closest results to real life. In the study, a Li-Ion battery cell was discharged using different electrical loads in order to perform the discharge process of the batteries of BEVs and HEVs in the battery SoC estimation in a way that best fits the discharge profile. A programmable electrical load device was used to generate different electrical loads. Considering the battery discharge profiles of BEVs and HEVs, the electrical load device was programmed at $1 \Omega, 2 \Omega, 3 \Omega, 4 \Omega, 5 \Omega, 6 \Omega, 7 \Omega, 8 \Omega, 9 \Omega$ and $10 \Omega$ resistance values and Discharges were repeated over the battery. In each discharge experiment, the OCV, current and temperature parameters of the Li-Ion battery were measured in real-time. After the discharge process, the battery was recharged and prepared for the next discharge experiment. Figure 4 shows the flow diagram of the method designed during the experiments.

During the battery discharge experiments, a main control card is designed to perform the discharge and charging process synchronously, to process and record the measurement data obtained from the sensors during the experimental processes, and to send them to the computer. Thanks to the main control card produced, the battery cell temperature, voltage and current parameters were measured and processed with the help of sensors on the card. The processed measurement value was recorded in the SD memory card.

Since all of the experiments were carried out on a fully charged LiIon battery cell, the direct SoC measurement method was applied with the current sensor connected on this card, and the battery was calculated and recorded on the SD memory card in the experiments. The technical characteristics of the battery cell used in the discharge experiments are given in Table 1, and the charge and discharge curves of the battery cell are given in Figure 5. In the experimental studies, the current, temperature and voltage parameters of the battery cell were measured and the battery SoC was performed by the Coulomb counting method, which is in the direct measurement methods class. Battery SoC estimation with Coulomb Counting method is easy and simplier than other methods. The biggest disadvantage of this method is that the initial capacity of the battery cannot be predicted.

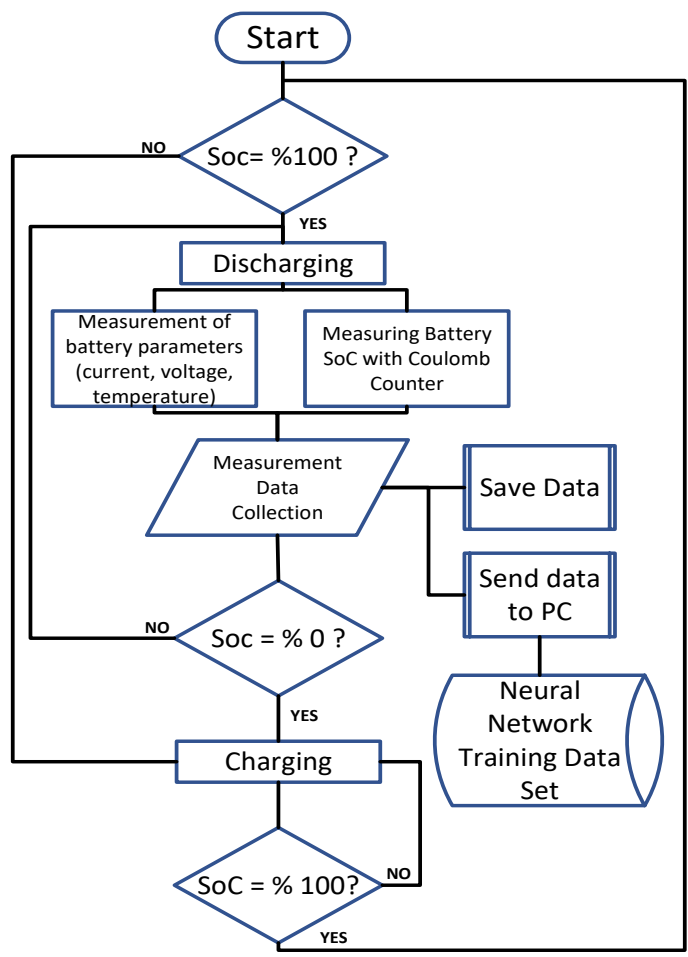

Fig. 4. Flow diagram of battery discharge experiments

Table 1. Panasonic NCR18650B Li-Ion battery cell specifications

\begin{tabular}{lcc}
\hline Parameter, Unit & Value \\
\hline Nominal capacity, mAh & 3.200 \\
\hline Nominal voltage, V & 3.6 \\
\hline Charging time, hour & 4 \\
\hline Weight, g & & 48.5 \\
\hline Temperature, ${ }^{\circ} \mathrm{C}$ & Charging & $0 \ldots 45$ \\
\cline { 2 - 3 } Discharging & $-20 \ldots 60$ \\
\hline Energy density, W/kg & & 243 \\
\hline
\end{tabular}

However, in experimental studies, the battery being in a fully charged state eliminates this disadvantage [24]. Therefore, by measuring and counting the battery discharge current and iteratively subtracting it from the nominal capacity of the battery, the battery SoC can be easily calculated. The mathematical expression of the Coulomb Counting method is given in Eq. (3).

$S o C_{t}=S o C_{t-1}+\left(\frac{1}{C_{N}}\right) \int_{t-1}^{t} \eta I_{b a t} d t$

In Equation 3, So $C_{t}$ is the SoC of the battery at time t, $S o C_{t-1}$ is the $\mathrm{SoC}$ of the battery at $\mathrm{t}-1, C_{N}$ is the nominal capacity of the battery, $\eta$ Coulomb efficiency coefficient $(\eta=1$ at the time of battery discharge, $\eta$ <at the time of battery charge. 1), $I_{b a t}$ represents battery current, $t$ represents sampling time. Figure 6 shows the working block diagram of the experimental setup used in experimental studies. The current measurements of the Panasonic NCR18650B Li-Ion battery cell used in the discharge experiments in the experimental setup were made using the LEM brand LTS25np current sensor. Thanks to the different connection configurations on the LTS25np, discharge current can be measured between $\pm 80 \mathrm{~A}$ and \pm 8 with high precision and accuracy. LEM brand LV 25 p voltage sensor is used for OCV measurement. Using the LV25p, the voltage between $10 \mathrm{~V}$ and $500 \mathrm{~V}$ is measured with high precision and accuracy. 


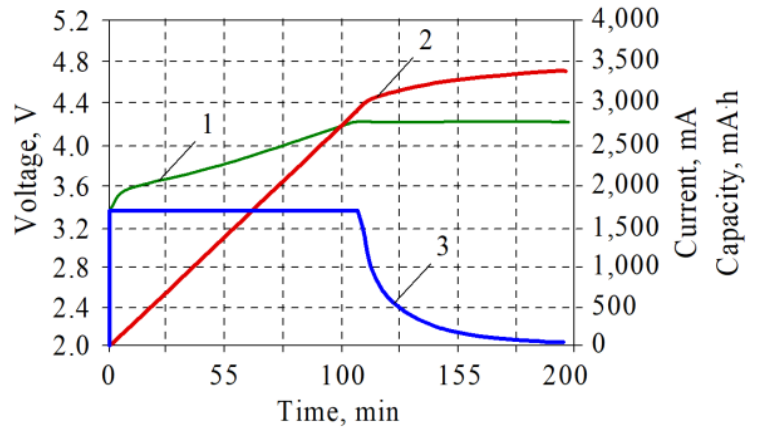

(a)

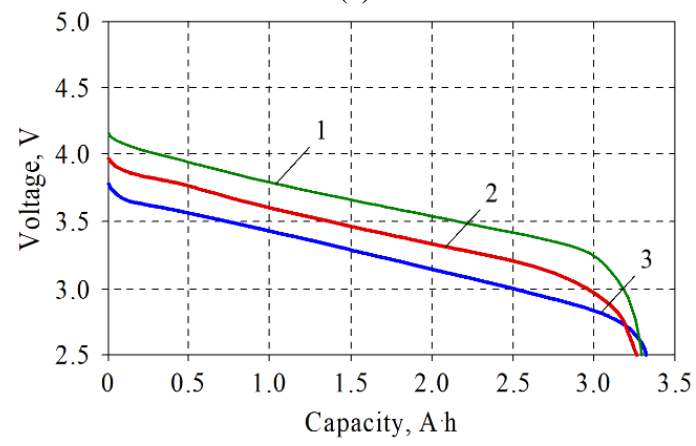

(b)

Fig. 5. Panasonic NCR18650B Li-Ion battery cell charge and discharge profiles [61], (a) Charge profile (1- voltage, 2- capacity, 3- current), (b) Discharge profile (1- 0.65 A, 2-3.2 A, 3- 6.5 A)
The LV25p transducer requires a power supply that supplies symmetrical voltage to operate. Therefore, in the study, a muRata brand NMH1205SC-2W PCB type DC-DC power supply was used. Thanks to this product, $5 \mathrm{~V}$ input voltage is converted to \pm $12 \mathrm{~V}$ output voltage. In addition, since the fully charged voltage of the battery is $4.2 \mathrm{~V}$, OCV cannot be measured with the voltage sensor in this range. Therefore, the OCV signal was increased using the Op-Amp circuit known as gain amplifier and OCV was measured. The temperature of the battery cell was measured using the Melexis brand MLX90615 digital infrared temperature sensor. The temperature sensor can measure between -40 and +115 degrees with a resolution of 0.02 degrees. The temperature of the battery cell was measured accurately in the experimental setup due to its high measurement sensitivity and non-contact measurement capability with infrared technology. The data obtained from the sensors in the battery cell was processed on the Arduino Mega 2560 microcontroller card, recorded on the SD memory card and transferred to the computer via the UART protocol. Arduino Mega 2560 is a development board with 54 Input / Output (I / O), 16 analog inputs and 16 Pulse Width Modulation (PWM) ports on the microcontroller board. Today, it is widely used in R\&D and academic studies. Liquid Crystal Display (LCD) was used to monitor the sensor data in the experiments and the test processes were monitored on the card in real-time. In battery discharge experiments, ARRAY brand 3711A programmable DC load device was used to load the battery cell.

Li-Ion Battery Charge and Discharge Experimental Setup

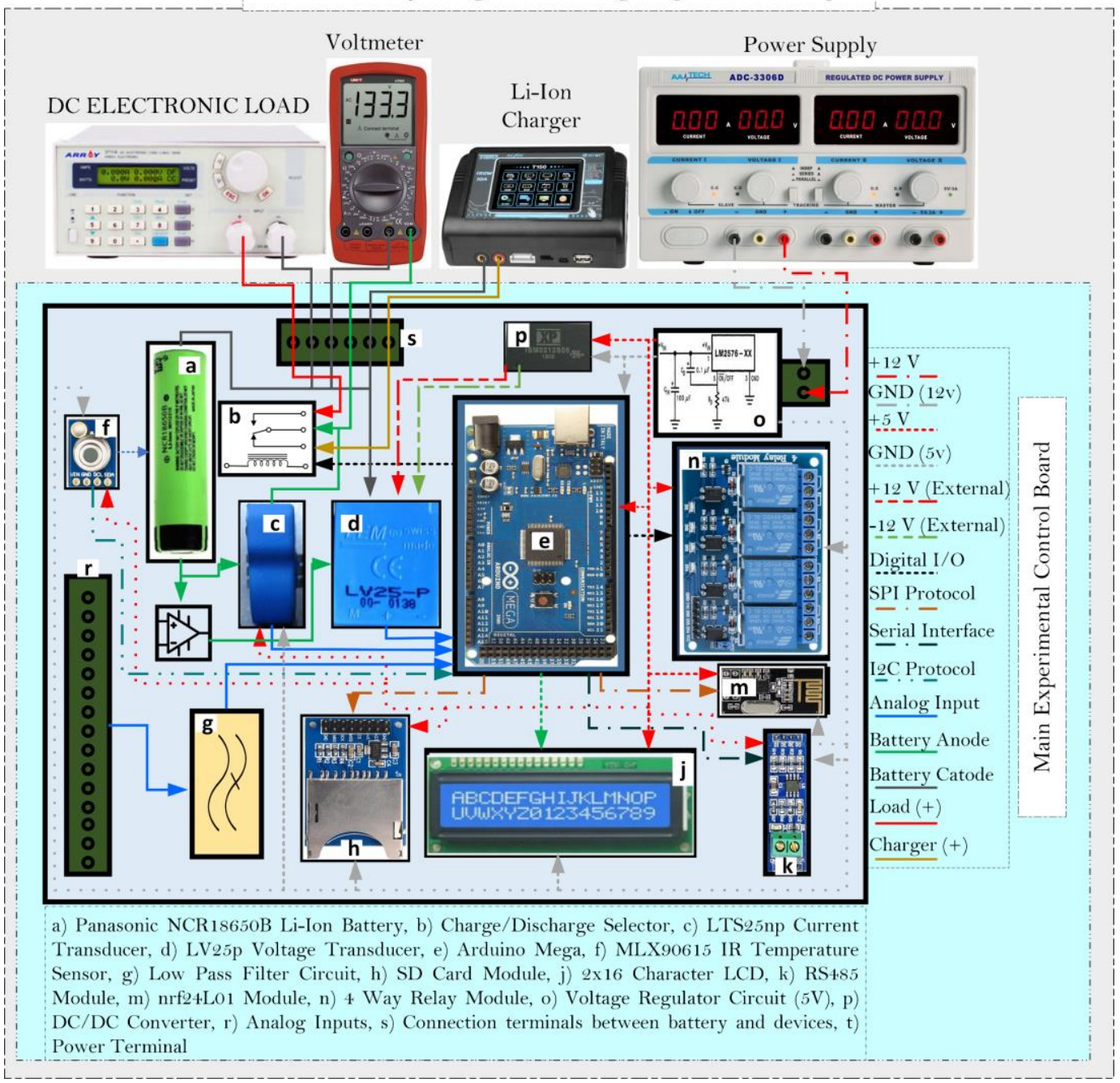

Fig. 6. Working block diagram of experimental setup 


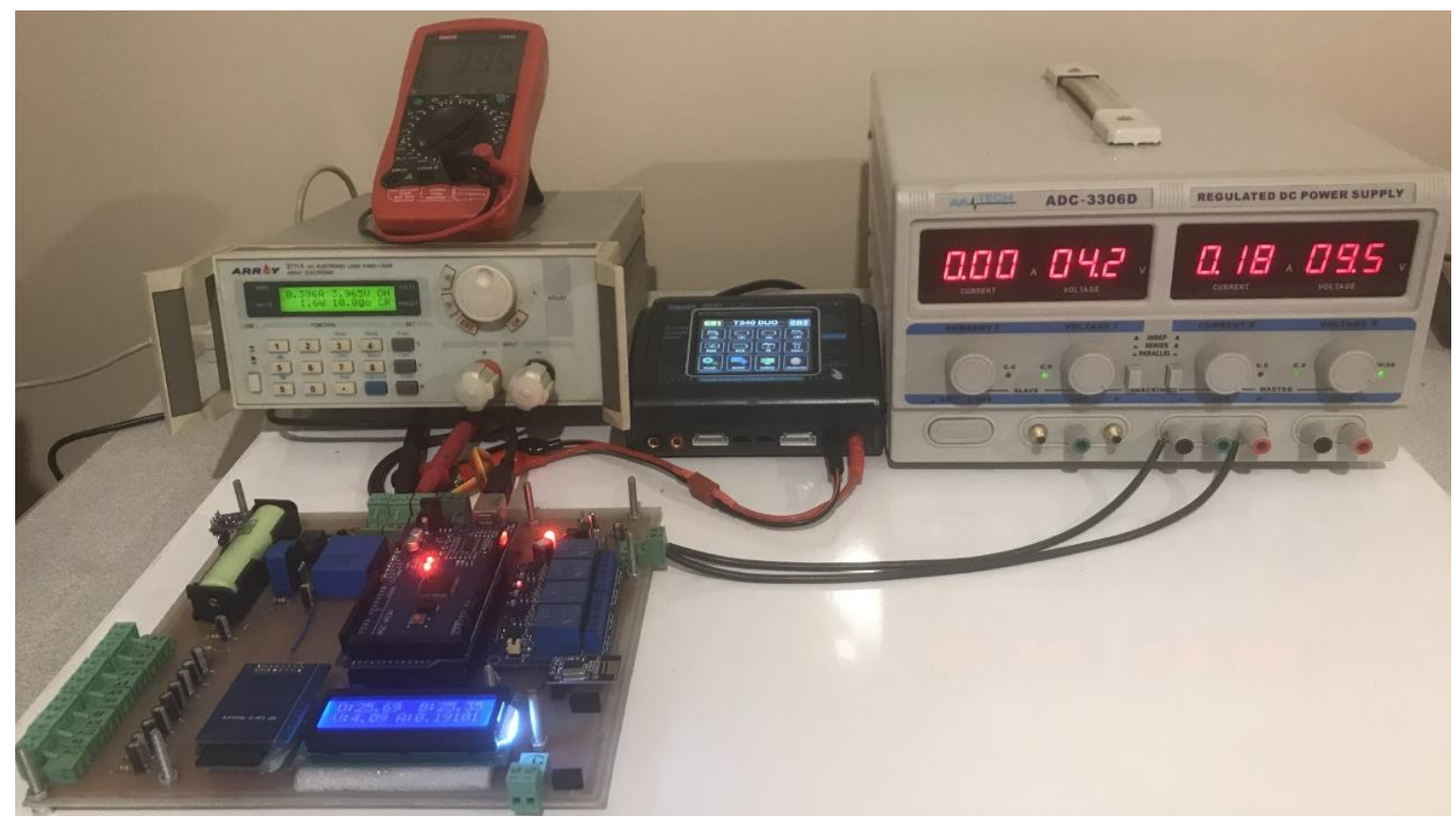

Fig. 7. Battery discharge experimental setup

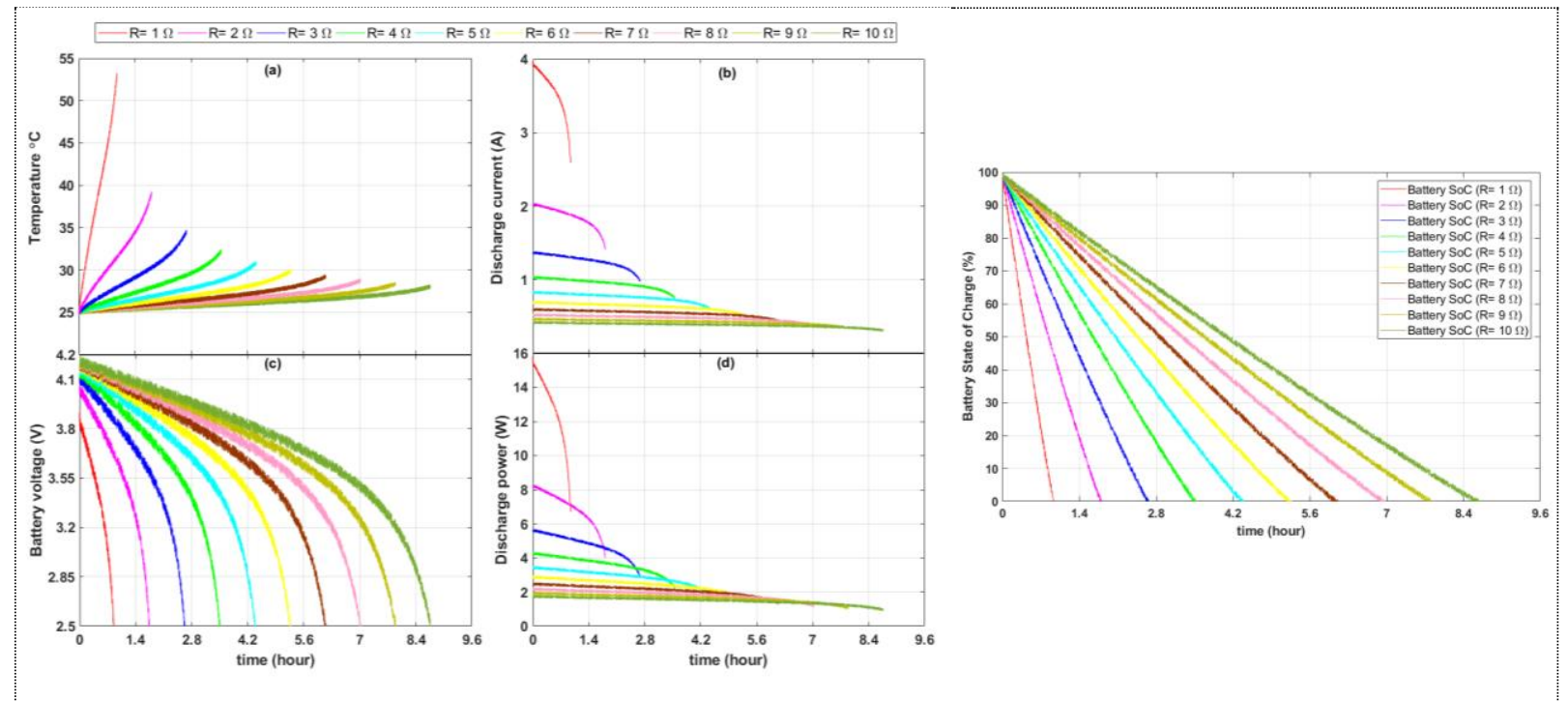

Fig. 8. The data obtained from the battery discharge experiments, a) battery temperature $(\mathrm{R}=1-10 \Omega)$, b) Battery discharge current $(\mathrm{R}=1-10 \Omega)$, c) Battery voltage $(\mathrm{R}=1-10 \Omega)$, d) Battery discharge power $(\mathrm{R}=1-10 \Omega)$, e) Battery SoC $(\mathrm{R}=1-10 \Omega)$

Thanks to the load device, it can be loaded up to $300 \mathrm{~W}$ power values and current, power and electrical load can be adjusted with $0.2 \%$ accuracy. The main control card is powered by an AATech brand ADC3306D model external AC / DC power supply. In the process of charging the battery cell after each discharge experiment, HTRC brand T240 Duo model battery charger with $240 \mathrm{~W}$ charging power was used. The visual of the experimental setup is given int he Figure 7. To be used in experimental studies, the sampling time for each parameter was determined as one second. Parameter measurement data set is obtained with ten different discharge experiments consists of $\cong 173 \mathrm{~K}$ data. Of the data set, $70 \%(\cong 121 \mathrm{~K})$ and $30 \%(\cong 49 \mathrm{~K})$ were used for training and testing purposes of network, respectively.

The experimental results of the battery temperature, voltage, current parameters, calculated instantaneous power and battery SoC parameters obtained from sensor measurements are given in the Figure 8.

\subsection{Training of Multi-layer Perceptron}

Training of the network was carried out by applying training data obtained from battery discharge experiments on MLP using Neural
Network Toolbox in Matlab environment. MLP modeled on Matlab-Neural Network Toolbox consists of 4 input parameters and one output parameter. MLP input parameters represent the battery temperature, voltage, discharge current and electrical power parameters obtained from discharge experiments. MLP output parameter represents the battery SoC obtained by Coulomb Counting method from the discharge experiments. In the modeled MLP structure, there are two separate hidden layers consisting of "8" and "4" neuron cells, respectively. BP algorithm was used in training the network. The tangent hyperbolic sigmoid "Tansig" function given in Equation 1 was used as the activation function in each nerve cell in the MLP. Network structure modeled on MatlabNeural Network Toolbox is given in Figure 9(a). In Figure 9(b) and Figure 9(c), MLP training results are given. The input parameters obtained from the battery discharge experiments were used in training the network at 1000 iterations according to the MLP target parameter. The success performance of MLP training was measured by the Mean Square Error (MSE) method. As can be understood from Figure 9(b), the MSE value of the network has decreased to 0.52198 at the end of the training. In Figure 9(c), the regression graphs obtained as a result of MLP training are given. 


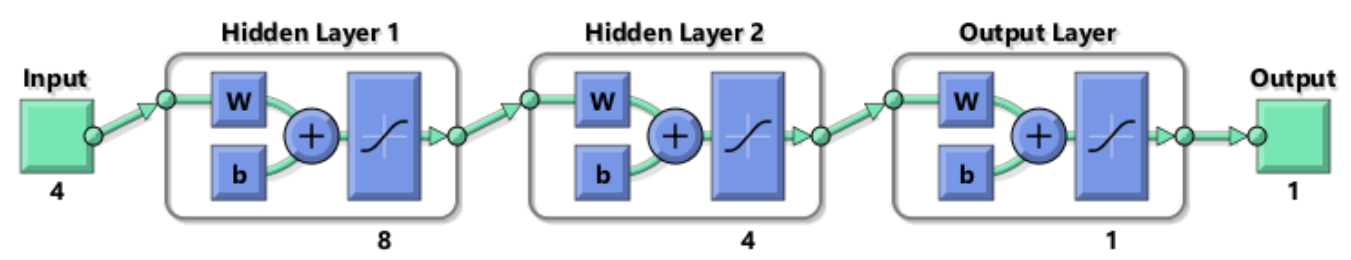

(a)

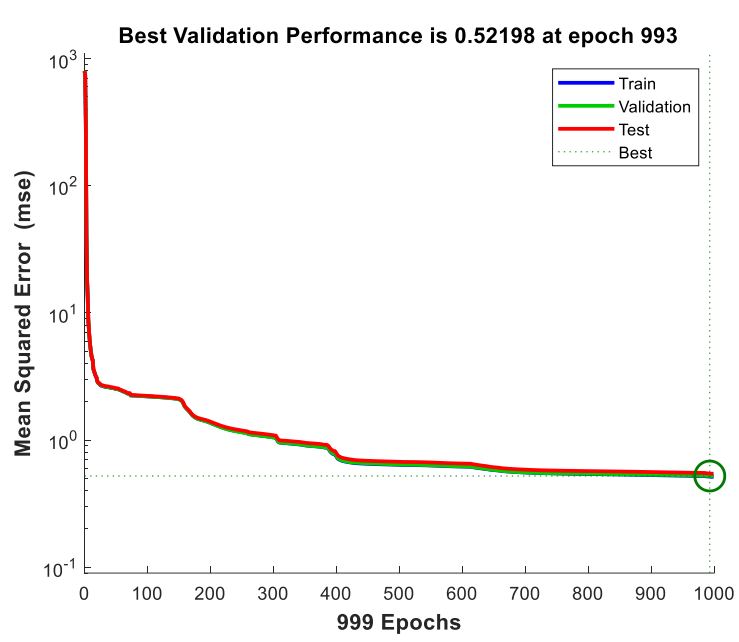

(b)
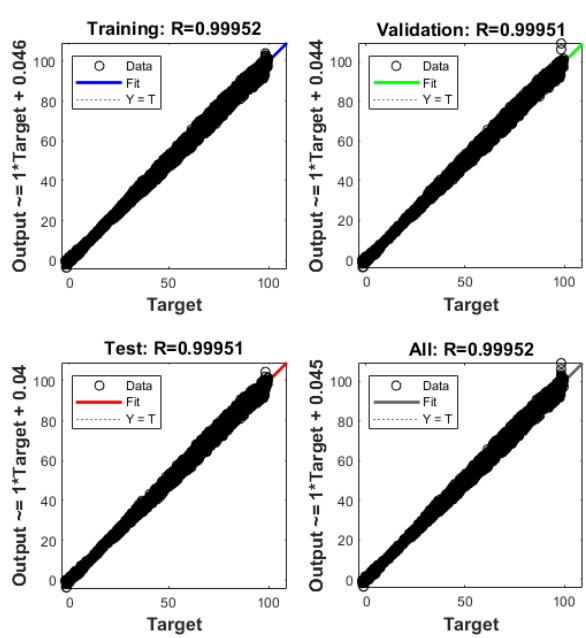

(c)

Fig. 9. MLP on Matlab-Neural Network Toolbox, a) Modeled MLP structure, b) MLP Training performance graph, c) MLP training regression graphs

Regression graphics are evaluated in the training, testing processes of the network, it is seen that the data are collected on the regression curve. Thus, training of the network has been successfully provided.

\section{Real-time Battery SoC Estimation}

In this part of the article, using the data obtained from the battery discharge experiments, the SoC was estimated in real-time with the trained MLP. Matlab/Simulink Arduino Real-time Toolbox has been used to estimate the battery SoC in real-time. ARRAY brand 3711A DC electronic load device used in the experiments for realtime SoC estimation is programmed according to different discharge current profiles. The $\mathrm{OCV}$, temperature and current parameters of the battery cell discharged with different discharge currents were measured by using sensors and the raw sensor data were transferred to the computer via UART protocol. The raw data transferred to the computer in real-time was imported to the Matlab environment with Arduino Real-time Toolbox and processed using various Simulink blocks. Battery current, OCV and temperature parameters were obtained from the processed raw sensor data on Matlab/Simulink via mathematical calculations. In addition, the real SoC of the battery and the electrical power consumption in the battery were calculated over the OCV and current parameters using the Coulomb Counting method over the battery current parameter, respectively. The data obtained from Matlab/Simulink were applied to the trained MLP in real-time. The battery SoC is estimated in real-time in different discharge current profiles until the battery is completely discharged. The working block diagram of the battery SoC estimation experiments is given in Figure 11 (a), and the Simulink software blocks of the real-time SoC estimation are given in Figure 11 (b). For real-time battery SoC estimation, three separate discharge current profiles were programmed in the DC electronic load device and were applied to the fully charged battery on experiment, respectively in three times. The discharge current profiles applied to the battery cell in real-time SoC estimation experiments are given in Figure 10.

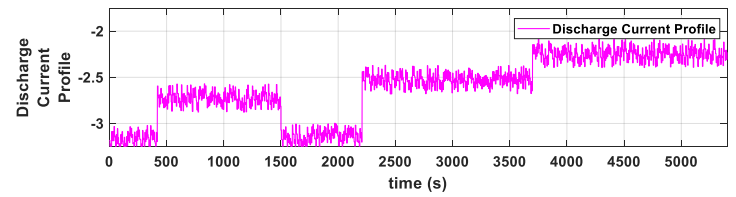

(a)

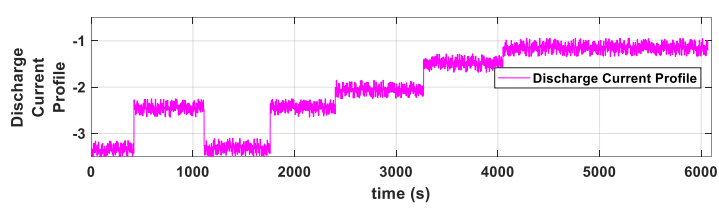

(b)

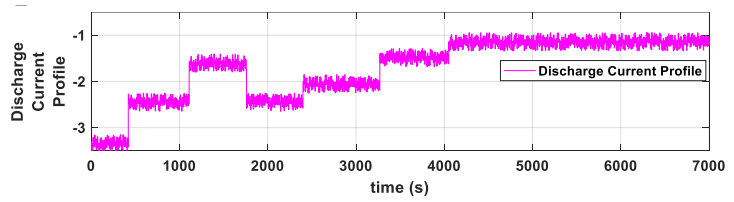

(c)

Fig. 10. Discharge current profiles, a) Profile-1, b) Profile-2, c) Profile-3 In order to better observe the estimation accuracy of the MLP modeled for real-time battery SoC estimation, three different discharge current profiles were selected. The data used in MLP training were created by using fixed resistors in discharge experiments. Undoubtedly, if SoC estimation experiments were made in the same discharge profiles, the prediction accuracy would be high and error rate would be low in the SoC estimation. However, considering that batteries used in systems with many dynamic variables such as BEVs and HEVs may have irregular discharge profiles, it is thought that it is useful to use variable discharge profiles in order to analyze the MLP performance in battery SoC estimation. 


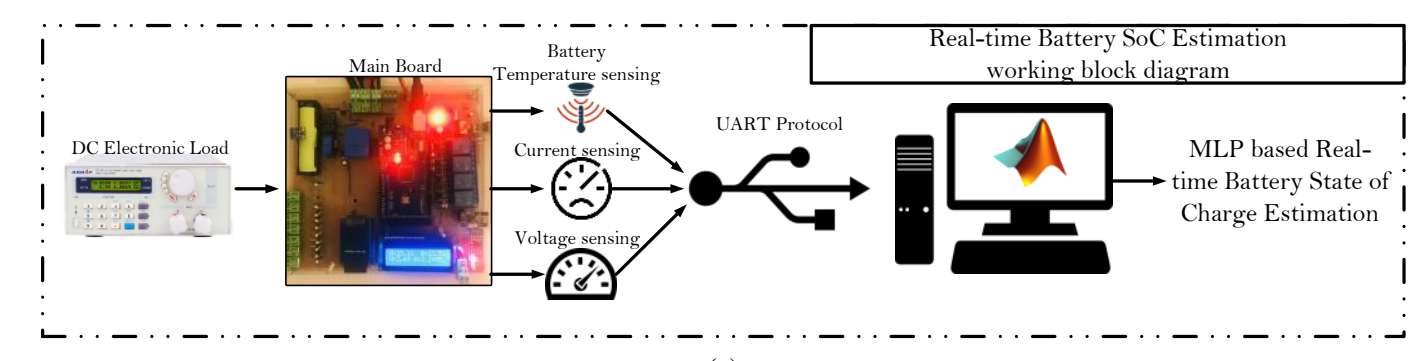

(a)

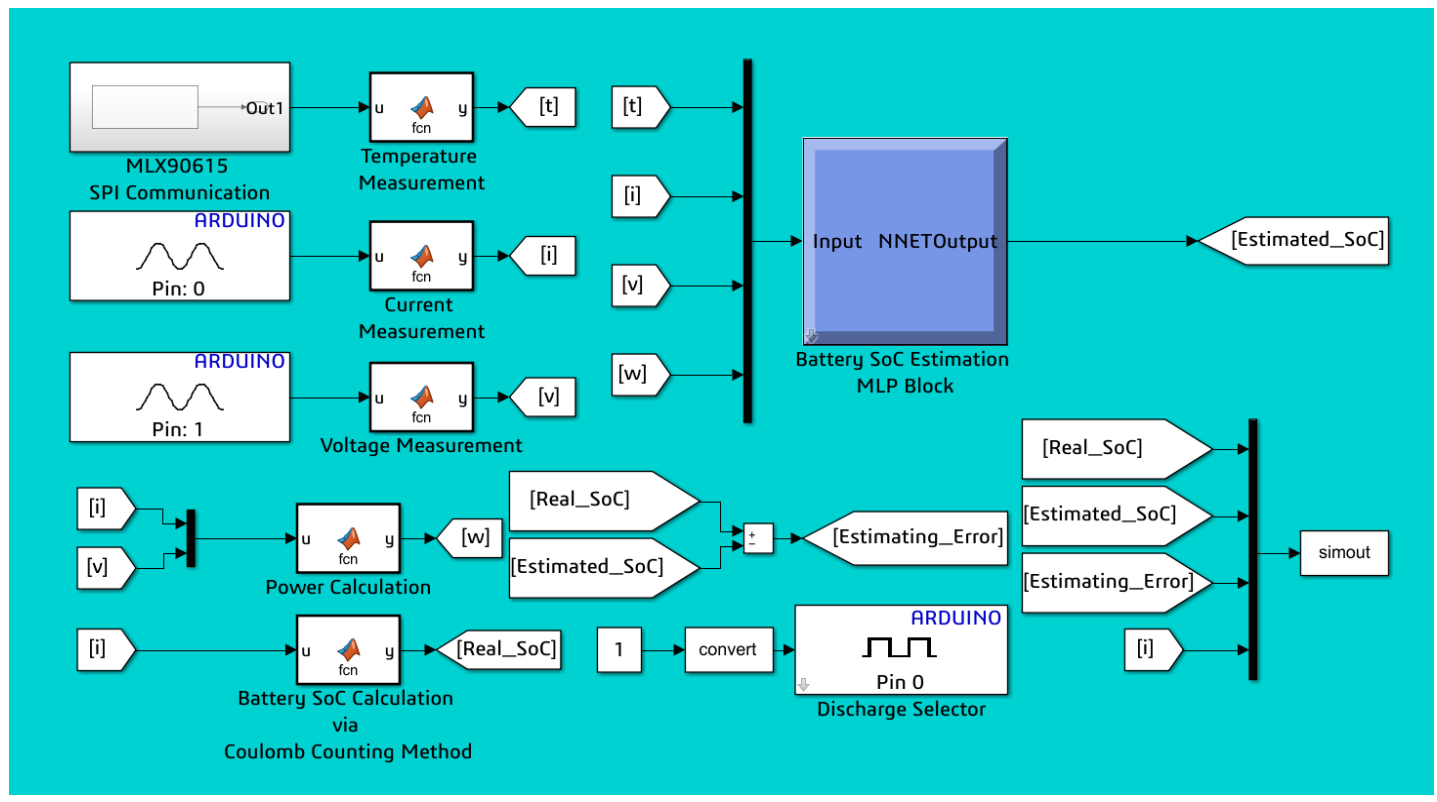

(b)

Fig. 11. Real-time battery SoC estimation, a) Experimental setup working block diagram, b) Matlab/Simulink software blocks.

Therefore, variable current profiles were chosen instead of fixed resistance profiles in order to better observe the success and prediction ability of MLP in real-time battery SoC estimation experiments. Thus, diversity is provided between the battery parameters measured in the SoC estimation experiments and the MLP training data. According to the discharge profiles given in Figure 10, the battery discharge process in Figure 10 (a) takes a shorter time compared to the profiles given in Figure 10 (b). The battery discharge process in Figure 10 (b) takes a shorter time compared to the profiles given in Figure 10 (c). MLP battery SoC estimation results and estimation errors were obtained from the experiments performed in three different discharge profiles. Battery SoC estimation results obtained from real-time experiments are given in Figure 12.

\section{Results and Discussions}

Li-Ion batteries are the most widely used battery type in BEVs and HEVs due to their durable and safe structure, high energy density, long life and light weight. Charging and discharging Li-Ion batteries within their capacity ranges is extremely important for the health of these batteries. Over charging and discharging of Li-Ion batteries cause their break down. In batteries, the SoC is the most important parameter used to protect the health of the battery and protect the battery from overcharging and discharging situations. The complex chemical structure of the batteries makes the battery SoC is cannot measure. Today, many researchers use various methods to accurately predict the battery SoC and try to obtain results closest to the real SoC of the battery. In this study, the state of charge of a Li-Ion battery cell was estimated in real-time with an MLP. MLP training data were obtained from a total of 10 separate discharge experiments performed using different resistance values ( $R=1-10$ ohms) as loads on an experiment set up for Li-Ion battery discharge and charging. Training data consists of battery current, temperature, OCV, discharge power consumption and SoC calculated by Coulomb Counting method. Designed in Matlab environment, MLP was trained with discharge experiment data and used in the real-time SoC estimation of the battery in experiments performed in variable discharge current profiles. Battery SoC is estimated in real-time in Matlab/Simulink environment. Here, the battery parameter was measured on the hardware, the measured parameters were imported to the Matlab environment from the hardware, and the battery charge status was estimated in real-time with the hardware-in-the-loop platform model in the cycle. Battery parameters measured in real-time experiments conducted in different discharge current profiles, calculated state of charge and charge state data estimated with MLP were recorded on Matlab Workspace. Results obtained from experimental studies were evaluated and discussed. Below are the results and discussions obtained in items.

- With MLP, the SoC of Li-Ion batteries can be estimated successfully. In the study, in order to better emphasize the success of the network, the battery parameters obtained from the discharge experiments performed under different resistances were used in the training of the network. However, Hardware-inthe-loop based real-time SoC estimation with the trained network was used in the battery parameters measured in different discharge current profiles. Although the data used for training the network and the data used in the performance tests of the network differ, the charge state estimation has a prediction error of at most $\pm 10 \%$. Therefore, it can be said that the battery SoC estimation with the MLP is successful. 


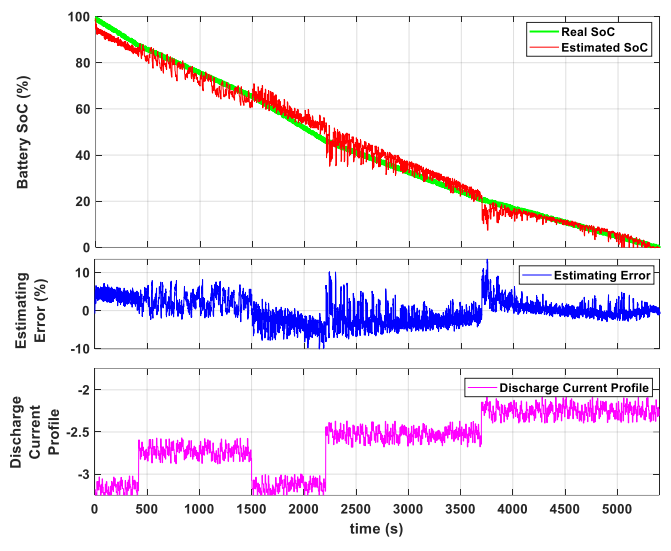

(a)

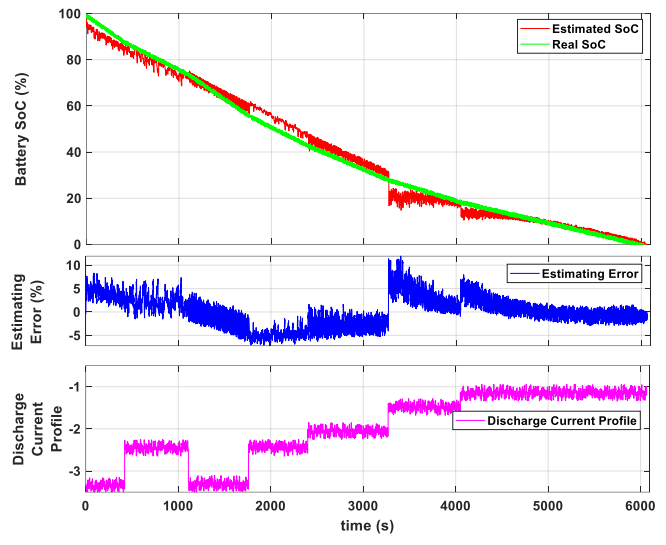

(b)

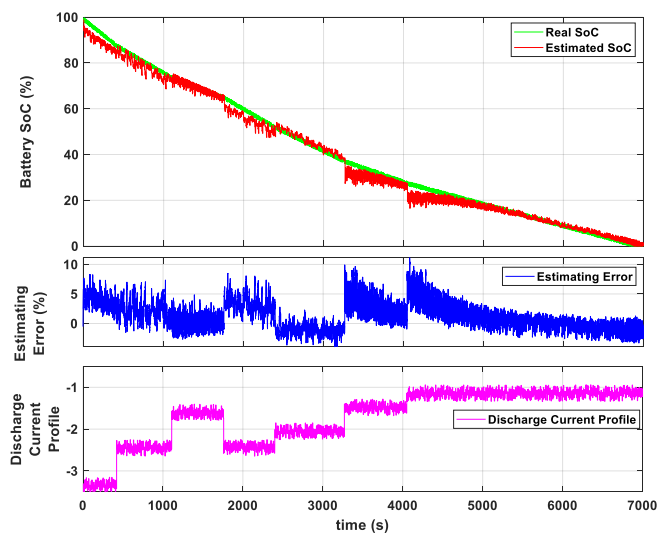

(c)

Fig. 12. Real-time battery SoC estimation results

- Coulomb Counting method, which is one of the direct SoC calculation methods, is a simple, computationally easy and inexpensive method. In the study, it was used to determine the actual SoC and the MLP performance was compared with the SoC parameter calculated with Coulomb Counting method. However, in order to get successful and accurate results in this method, it should be known how much capacity of battery is initially. At this point, MLP be different from direct calculation methods such as Coulomb Counting method because of their data-driven working principles and their trainability. Thus, it does not need the initial capacity value information as in the Coulomb Counting method.

- Although successful predictions have been made with the MLP in the study, data is needed for the training and the closest simulation of the network. Missing or insufficient training data of the network will result in inaccurate estimation results. In addition, SoC estimation with MLP model approaches does not make SoC estimation possible in other battery types, since it will take the data that the network is trained as a sample. It is necessary to prepare training data and retraining the network for different batteries whose SoC will be estimated. This will cause the process to be long time. In addition, the aging factor of the battery is an important parameter. Since it is known that the capacity of batteries will decrease as they age, it is predicted that errors in the estimation results will increase as the battery ages.

- Another important topic in estimating the battery SoC with the MLP approach is the sensor and control equipment used in the experiments. In order to increase the accuracy of the results, current, voltage and temperature measurements should be highly accurate and precise. For this, measuring range wide current sensor and high precision Analog Digital Converter (ADC) are required. Therefore, sensors with high accuracy and sensitivity were used in the study.

SoC estimation in Li-Ion batteries is a developing issue that keeps up to date. Therefore, it is anticipated that the literature will be spread with the studies to be carried out in this field and developed with the proposed new methods. Today, new methods are being developed, discussed and shared in this field day by day.

\section{References}

[1] J.-R. Riba, C. López-Torres, L. Romeral, and A. Garcia, "Rare-earthfree propulsion motors for electric vehicles: A technology review," Renew. Sustain. Energy Rev., vol. 57, pp. 367-379, 2016.

[2] A. Emadi, Y. J. Lee, and K. Rajashekara, "Power Electronics and Motor Drives in Electric, Hybrid Electric, and Plug-In Hybrid Electric Vehicles," IEEE Trans. Ind. Electron., vol. 55, no. 6, pp. 2237-2245, 2008.

[3] C. C. Chan, "The state of the art of electric and hybrid vehicles," Proc. IEEE, vol. 90, no. 2, pp. 247-275, 2002.

[4] J. A. P. Lopes, F. J. Soares, and P. M. R. Almeida, "Integration of Electric Vehicles in the Electric Power System," Proc. IEEE, vol. 99, no. 1, pp. 168-183, 2011.

[5] A. Poullikkas, "Sustainable options for electric vehicle technologies," Renew. Sustain. Energy Rev., vol. 41, pp. 1277-1287, 2015.

[6] S. Ould Amrouche, D. Rekioua, T. Rekioua, and S. Bacha, "Overview of energy storage in renewable energy systems," Int. J. Hydrogen Energy, vol. 41, no. 45, pp. 20914-20927, 2016.

[7] C. Capasso and O. Veneri, "Experimental analysis on the performance of lithium based batteries for road full electric and hybrid vehicles," 
Appl. Energy, vol. 136, pp. 921-930, 2014.

[8] A. F. Moghaddam, M. Mnati, H. Sun, and A. V d. Bossche, "Electric Vehicles Charging Concepts for Lithium Based Batteries," in 2018 7th International Conference on Renewable Energy Research and Applications (ICRERA), 2018, pp. 397-401.

[9] S. M. A. S. Bukhari, J. Maqsood, M. Q. Baig, S. Ashraf, and T. A. Khan, "Comparison of Characteristics -- Lead Acid, Nickel Based, Lead Crystal and Lithium Based Batteries," in 2015 17th UKSimAMSS International Conference on Modelling and Simulation (UKSim), 2015, pp. 444-450.

[10] V. Pop, H. J. Bergveld, D. Danilov, P. P. L. Regtien, and P. H. L. Notten, "State-of-the-art of battery state-of-charge determination," Batter. Manag. Syst. Accurate State-of-Charge Indic. Batter. Appl., pp. 11-45, 2008.

[11] S. Pang, J. Farrell, J. Du, and M. Barth, "Battery state-of-charge estimation," in Proceedings of the 2001 American control conference.(Cat. No. 01CH37148), 2001, vol. 2, pp. 1644-1649.

[12]M. A. Hannan, M. S. H. Lipu, A. Hussain, and A. Mohamed, "A review of lithium-ion battery state of charge estimation and management system in electric vehicle applications: Challenges and recommendations," Renew. Sustain. Energy Rev., vol. 78, pp. 834 $854,2017$.

[13]Z. Chen, L. Yang, X. Zhao, Y. Wang, and Z. He, "Online state of charge estimation of Li-ion battery based on an improved unscented Kalman filter approach,” Appl. Math. Model., vol. 70, pp. 532-544, 2019.

[14]M. Mastali, J. Vazquez-Arenas, R. Fraser, M. Fowler, S. Afshar, and M. Stevens, "Battery state of the charge estimation using Kalman filtering," J. Power Sources, vol. 239, pp. 294-307, 2013.

[15]J. Sabatier, M. Aoun, A. Oustaloup, G. Grégoire, F. Ragot, and P. Roy, "Fractional system identification for lead acid battery state of charge estimation," Signal Processing, vol. 86, no. 10, pp. 2645-2657, 2006.

[16]R. Xiong, J. Cao, Q. Yu, H. He, and F. Sun, "Critical Review on the Battery State of Charge Estimation Methods for Electric Vehicles," IEEE Access, vol. 6, pp. 1832-1843, 2018.

[17]M. Charkhgard and M. Farrokhi, "State-of-Charge Estimation for Lithium-Ion Batteries Using Neural Networks and EKF," IEEE Trans. Ind. Electron., vol. 57, no. 12, pp. 4178-4187, 2010.

[18]F. Sun, X. Hu, Y. Zou, and S. Li, "Adaptive unscented Kalman filtering for state of charge estimation of a lithium-ion battery for electric vehicles," Energy, vol. 36, no. 5, pp. 3531-3540, 2011.

[19]I.-S. Kim, "The novel state of charge estimation method for lithium battery using sliding mode observer," J. Power Sources, vol. 163, no. 1, pp. 584-590, 2006.

[20]K. S. Ng, C.-S. Moo, Y.-P. Chen, and Y.-C. Hsieh, "Enhanced coulomb counting method for estimating state-of-charge and state-ofhealth of lithium-ion batteries," Appl. Energy, vol. 86, no. 9, pp. 1506$1511,2009$.

[21]K. Ng, Y. Huang, C. Moo, and Y. Hsieh, “An enhanced coulomb counting method for estimating state-of-charge and state-of-health of lead-acid batteries," in INTELEC 2009 - 31st International Telecommunications Energy Conference, 2009, pp. 1-5.

[22] Y. Jeong, Y. Cho, J. Ahn, S. Ryu, and B. Lee, "Enhanced Coulomb counting method with adaptive SOC reset time for estimating OCV,' in 2014 IEEE Energy Conversion Congress and Exposition (ECCE), 2014, pp. 1313-1318.

[23] M. A. Awadallah and B. Venkatesh, "Accuracy improvement of SOC estimation in lithium-ion batteries," J. Energy Storage, vol. 6, pp. 95 $104,2016$.

[24]E. Leksono, I. N. Haq, M. Iqbal, F. X. N. Soelami, and I. G. N Merthayasa, "State of charge (SoC) estimation on LiFePO4 battery module using Coulomb counting methods with modified Peukert," in 2013 Joint International Conference on Rural Information Communication Technology and Electric-Vehicle Technology (rICT ICeV-T), 2013, pp. 1-4.

[25]L. Zhao, M. Lin, and Y. Chen, "Least-squares based coulomb counting method and its application for state-of-charge (SOC) estimation in electric vehicles,” Int. J. Energy Res., vol. 40, no. 10, pp. 1389-1399, 2016.

[26]Li Ran, Wu Junfeng, Wang Haiying, and Li Gechen, "Prediction of state of charge of Lithium-ion rechargeable battery with electrochemical impedance spectroscopy theory," in 2010 5th IEEE Conference on Industrial Electronics and Applications, 2010, pp. 684 688.

[27]K. Bundy, M. Karlsson, G. Lindbergh, and A. Lundqvist, "An electrochemical impedance spectroscopy method for prediction of the state of charge of a nickel-metal hydride battery at open circuit and during discharge," J. Power Sources, vol. 72, no. 2, pp. 118-125, 1998

[28]I. A. J. Gordon et al., "Electrochemical Impedance Spectroscopy response study of a commercial graphite-based negative electrode for Li-ion batteries as function of the cell state of charge and ageing," Electrochim. Acta, vol. 223, pp. 63-73, 2017.

[29]D. Andre, M. Meiler, K. Steiner, C. Wimmer, T. Soczka-Guth, and D. U. Sauer, "Characterization of high-power lithium-ion batteries by electrochemical impedance spectroscopy. I. Experimental investigation," J. Power Sources, vol. 196, no. 12, pp. 5334-5341, 2011.

[30]U. Westerhoff, T. Kroker, K. Kurbach, and M. Kurrat, "Electrochemical impedance spectroscopy based estimation of the state of charge of lithium-ion batteries," J. Energy Storage, vol. 8, pp. 244-256, 2016.

[31]Q. Wang, Y. He, J. Shen, X. Hu, and Z. Ma, "State of ChargeDependent Polynomial Equivalent Circuit Modeling for Electrochemical Impedance Spectroscopy of Lithium-Ion Batteries,' IEEE Trans. Power Electron., vol. 33, no. 10, pp. 8449-8460, 2018.

[32] S. Lee, J. Kim, J. Lee, and B. H. Cho, "State-of-charge and capacity estimation of lithium-ion battery using a new open-circuit voltage versus state-of-charge," J. Power Sources, vol. 185, no. 2, pp. $1367-$ 1373,2008

[33] Y. Xing, W. He, M. Pecht, and K. L. Tsui, "State of charge estimation of lithium-ion batteries using the open-circuit voltage at various ambient temperatures," Appl. Energy, vol. 113, pp. 106-115, 2014.

[34]R. Xiong, Q. Yu, L. Y. Wang, and C. Lin, “A novel method to obtain the open circuit voltage for the state of charge of lithium ion batteries in electric vehicles by using H infinity filter," Appl. Energy, vol. 207, pp. 346-353, 2017.

[35]C. Weng, J. Sun, and H. Peng, "A unified open-circuit-voltage model of lithium-ion batteries for state-of-charge estimation and state-ofhealth monitoring," J. Power Sources, vol. 258, pp. 228-237, 2014.

[36] S. Tong, M. P. Klein, and J. W. Park, "On-line optimization of battery open circuit voltage for improved state-of-charge and state-of-health estimation," J. Power Sources, vol. 293, pp. 416-428, 2015.

[37] Y. Zheng, M. Ouyang, X. Han, L. Lu, and J. Li, "Investigating the error sources of the online state of charge estimation methods for lithiumion batteries in electric vehicles," J. Power Sources, vol. 377, pp. 161188,2018

[38]S. Piller, M. Perrin, and A. Jossen, "Methods for state-of-charge determination and their applications," J. Power Sources, vol. 96, no. 1, pp. 113-120, 2001.

[39] Y. Tian, B. Xia, W. Sun, Z. Xu, and W. Zheng, "A modified model based state of charge estimation of power lithium-ion batteries using unscented Kalman filter," J. Power Sources, vol. 270, pp. 619-626, 2014

[40]M. Corno, N. Bhatt, S. M. Savaresi, and M. Verhaegen, "Electrochemical Model-Based State of Charge Estimation for Li-Ion Cells," IEEE Trans. Control Syst. Technol., vol. 23, no. 1, pp. $117-$ $127,2015$.

[41]C. Zhang, K. Li, L. Pei, and C. Zhu, "An integrated approach for realtime model-based state-of-charge estimation of lithium-ion batteries," J. Power Sources, vol. 283, pp. 24-36, 2015.

[42]H. He, X. Zhang, R. Xiong, Y. Xu, and H. Guo, "Online model-based estimation of state-of-charge and open-circuit voltage of lithium-ion batteries in electric vehicles," Energy, vol. 39, no. 1, pp. 310-318, 2012

[43]L. Kang, X. Zhao, and J. Ma, "A new neural network model for the state-of-charge estimation in the battery degradation process," Appl. Energy, vol. 121, pp. 20-27, 2014.

[44]W. He, N. Williard, C. Chen, and M. Pecht, "State of charge estimation for Li-ion batteries using neural network modeling and unscented Kalman filter-based error cancellation," Int. J. Electr. Power Energy Syst., vol. 62, pp. 783-791, 2014.

[45]C. Bo, B. Zhifeng, and C. Binggang, "State of charge estimation based on evolutionary neural network," Energy Convers. Manag., vol. 49, no. 10, pp. 2788-2794, 2008.

[46]F. Yang, W. Li, C. Li, and Q. Miao, "State-of-charge estimation of lithium-ion batteries based on gated recurrent neural network," Energy, vol. 175, pp. 66-75, 2019.

[47] M. A. Hannan, M. S. H. Lipu, A. Hussain, M. H. Saad, and A. Ayob, "Neural Network Approach for Estimating State of Charge of LithiumIon Battery Using Backtracking Search Algorithm," IEEE Access, vol. 6, pp. 10069-10079, 2018

[48] W.-Y. Chang, "Estimation of the state of charge for a LFP battery using a hybrid method that combines a RBF neural network, an OLS algorithm and AGA," Int. J. Electr. Power Energy Syst., vol. 53, pp 603-611, 2013

[49]D. Liu, L. Li, Y. Song, L. Wu, and Y. Peng, "Hybrid state of charge estimation for lithium-ion battery under dynamic operating conditions,” Int. J. Electr. Power Energy Syst., vol. 110, pp. 48-61, 
2019.

[50] G. S. Misyris, D. I. Doukas, T. A. Papadopoulos, D. P. Labridis, and V. G. Agelidis, "State-of-Charge Estimation for Li-Ion Batteries: A More Accurate Hybrid Approach," IEEE Trans. Energy Convers., vol. 34, no. 1, pp. 109-119, 2019.

[51]J. P. Rivera-Barrera, N. Muñoz-Galeano, and H. O. SarmientoMaldonado, "SoC estimation for lithium-ion batteries: Review and future challenges," Electronics, vol. 6, no. 4, p. 102, 2017.

[52] J. Pablo Rivera-Barrera, N. Munoz-Galeano, and H. Omar SarmientoMaldonado, "SoC Estimation for Lithium-ion Batteries: Review and Future Challenges," ELECTRONICS, vol. 6, no. 4, 2017.

[53]Z. Chen, S. Qiu, M. A. Masrur, and Y. L. Murphey, "Battery state of charge estimation based on a combined model of Extended Kalman Filter and neural networks," in The 2011 International Joint Conference on Neural Networks, 2011, pp. 2156-2163.

[54] J. Gomez, R. Nelson, E. E. Kalu, M. H. Weatherspoon, and J. P. Zheng, "Equivalent circuit model parameters of a high-power Li-ion battery: Thermal and state of charge effects," J. Power Sources, vol. 196, no. 10, pp. 4826-4831, 2011.

[55]Y. Zhou, C. Bai, and J. Sun, "Application of Genetic Neural Network in Power Battery Charging State-of-Charge Estimation," Int. J. Intell. Syst. Appl., vol. 3, no. 2, p. 24, 2011.

[56]D. Jiménez-Bermejo, J. Fraile-Ardanuy, S. Castaño-Solis, J. Merino, and R. Álvaro-Hermana, "Using Dynamic Neural Networks for Battery State of Charge Estimation in Electric Vehicles," Procedia Comput. Sci., vol. 130, pp. 533-540, 2018.

[57]B. Yegnanarayana, Artificial neural networks. PHI Learning Pvt. Ltd., 2009.

[58] J. A. K. Suykens, J. P. L. Vandewalle, and B. L. de Moor, Artificial neural networks for modelling and control of non-linear systems. Springer Science \& Business Media, 2012.

[59]M. M. Mijwel, "Artificial neural networks advantages and disadvantages," Retrieved from LinkedIn https//www. linkedin. com/pulse/artificial-neuralnet Work., 2018.

[60] Xinghuo Yu, M. O. Efe, and O. Kaynak, "A general backpropagation algorithm for feedforward neural networks learning," IEEE Trans. Neural Networks, vol. 13, no. 1, pp. 251-254, 2002.

[61]O. Plakhtii, V. Nerubatskyi, A. Mashura, and D. Hordiienko, "The Analysis of Mathematical Models of Charge-Discharge Characteristics in Lithium-Ion Batteries," in 2020 IEEE 40th International Conference on Electronics and Nanotechnology (ELNANO), 2020, pp. 635-640. 